Article

\title{
Methyl Jasmonate Treatment of Broccoli Enhanced Glucosinolate Concentration, Which Was Retained after Boiling, Steaming, or Microwaving
}

\author{
Yu-Chun Chiu ${ }^{1}$, Kristen Matak ${ }^{2}$ and Kang-Mo Ku ${ }^{1,3, *}$ \\ 1 Division of Plant and Soil Sciences, West Virginia University, Morgantown, WV 26506, USA; \\ yuchiu@mix.wvu.edu \\ 2 Division of Animal and Nutritional Sciences, West Virginia University, Morgantown, WV 26506, USA; \\ Kristen.Matak@mail.wvu.edu \\ 3 Department of Horticulture, College of Agriculture and Life Sciences, Chonnam National University, \\ Gwangju 61886, Korea \\ * Correspondence: ku9@jnu.ac.kr; Tel.: +82-62-530-2065; Fax: +82-62-530-2069
}

Received: 26 May 2020; Accepted: 6 June 2020; Published: 8 June 2020

\begin{abstract}
Exogenous methyl jasmonate (MeJA) treatment was known to increase the levels of neoglucobrassicin and their bioactive hydrolysis products in broccoli (Brassica oleracea var. italica), but the fate of MeJA-induced glucosinolates (GSLs) after various cooking methods was unknown. This study measured the changes in GSLs and their hydrolysis compounds in broccoli treated with MeJA and the interaction between MeJA and cooking treatments. All cooked MeJA-treated broccoli contained significantly more GSLs than untreated broccoli $(p<0.05)$. After 5 min of cooking (boil, steam, microwave), MeJA-treated broccoli still contained 1.6- to 2.3-fold higher GSL content than untreated broccoli. Neoglucobrassicin hydrolysis products were also significantly greater in steamed and microwaved MeJA-treated broccoli. The results show that exogenous MeJA treatment increases neoglucobrassicin and its hydrolysis compounds in broccoli even after cooking. Once the positive and negative effects of these compounds are better understood, the results of this experiment can be a valuable tool to help food scientists, nutrition scientists, and dieticians determine how to incorporate raw or cooked broccoli and Brassica vegetables in the diet.
\end{abstract}

Keywords: broccoli; methyl jasmonate; glucosinolate; glucosinolate hydrolysis products; cooking

\section{Introduction}

Broccoli consumption (Brassica oleracea var. italica) is associated with anti-cancer activity in in-vitro and in-vivo trials, due to the high content of phytochemicals, minerals, vitamins, and fibers. GSLs are a group of phytochemicals in broccoli which have been studied extensively for their health benefits; for example, an inverse relationship has been reported between the risk of breast cancer in Caucasian women and consumption of broccoli [1]. Furthermore, consumption of broccoli was shown to reduce the concentration of hepatic triglycerides in mice, and long-term broccoli consumption may promote liver health [2].

In Brassica oleracea, aliphatic GSLs derived from methionine and isoleucine are known [3,4], and indole GSL can be derived from tryptophan [3]. In plants, GSL are hydrolyzed by an endogenous enzyme myrosinase (EC 3.2.3.1, thioglucoside glucohydrolase) when insects attack or a physical wound is formed. This reaction produces the corresponding hydrolysis products. For example, isothiocyanates (ITCs) are GSL hydrolysis products reported to possess generally higher bioactivity than other types of GSL hydrolysis products [5]. The chemopreventive effect of sulforaphane, a well-known ITC, is greater than other GSL hydrolysis products in cell culture assay [6], animal assay [7], and a clinical 
trial [1]. However, the accumulation of its precursor, glucoraphanin, is mainly determined by the genetic background of the plant rather than the environmental factors [8]; therefore, increasing its concentration through agricultural practices is limited. In contrast, indole GSL levels are mainly affected by environmental factors [8], which implies that the concentration of indole GSL can be increased by agricultural practices.

Broccoli is often eaten cooked, or sometimes eaten raw as an ingredient in salads. Several studies have investigated the impact of common cooking methods, including boiling, steaming, and microwaving, on the retention of GSL, with boiling generally causing substantial loss of GSL [9-11]. In addition to the GSL loss, cooking also inactivates myrosinase, the enzyme converting GSL into hydrolysis products, and then hinders the formation of hydrolysis products [12]. To date, methods of delivering cooked broccoli without losing its nutritional benefits are still lacking in the literature, although consuming cooked broccoli is the most common practice for consumers.

Exogenous methyl jasmonate (MeJA) application increases inducible indole GSLs (neoglucobrassicin and glucobrassicin) or benzenic GSLs (gluconasturtiin) concentrations in Brassica vegetables [13] due to its anti-repellent activity. MeJA is a volatile jasmonate derivative that responds to herbivore damage $[14,15]$, and GSL biosynthesis is upregulated by JA signaling transduction $[16,17]$. Applying exogenous MeJA triggers a defense response to insect damage in Brassica plants [18]; as a result, defense-related metabolites, such as GSLs, are upregulated [6] either by depletion of the carbon pool (glucose) or by the conserved transcriptional network [19]. MeJA application can be used as an agricultural practice to enhance the nutritional quality of broccoli. Indole-3-carbinol (I3C; derived from glucobrassicin) was associated with reducing the risk of hormone-responsive cancers [20] including breast [21] and prostate cancer [22]. N-methoxy-indole-3-carbinol (NMI3C), derived from neoglucobrassicin, was reported to reduce the growth of human colon cells by inhibiting CYP1A enzyme, and its potency was higher than I3C in vitro [23]. On the other hand, indole GSL was reported to have negative effects on health in previous literature [24,25]. It was shown that neoglucobrassicin is highly mutagenic [24], and the consumption of I3C (hydrolysis product of glucobrassicin) was suggested to have Janus properties, where I3C may promote carcinogenesis if it is ingested after the carcinogen in animal models [25]. Quantification of these compounds in raw and cooked Brassica crops may provide valuable information for nutrition scientists and dieticians once the effects of these compounds in humans becomes clearer.

Although MeJA treatment significantly increases GSL in broccoli, there has been limited study on myrosinase activity and the amount of GSL hydrolysis products in MeJA-treated broccoli after cooking. We hypothesized that MeJA-induced changes in broccoli may affect GSL degradation, myrosinase activity, or myrosinase-associated proteins during domestic cooking, resulting in different GSL or hydrolysis product compositions. As GSL hydrolysis compounds are the actual bioactive compounds rather than GSL itself [26], it is critical to determine whether MeJA treatment increases GSL hydrolysis products and myrosinase in cooked broccoli. Therefore, the major research questions of this study were to evaluate how MeJA application to broccoli plants will affect GSL concentration, myrosinase activity, GSL hydrolysis product amounts, and other metabolites in broccoli florets after boiling, steaming, and microwaving treatments.

\section{Materials and Methods}

\subsection{Broccoli Cultivation and Methyl Jasmonate Treatment}

'Green Magic' broccoli was selected for its consistent response to $250 \mu \mathrm{M}$ MeJA application year to year [27]. Seeds of 'Green Magic' broccoli were obtained from Johnny's Selected Seeds (Albion, ME, USA). Broccoli was grown from seeds in the cell pack (one plant per pack) filled with Sunshine \#1 Mix (Sun Gro Horticulture, Vancouver, BC, Canada) in the greenhouse (Morgantown, WV, USA). The seedlings were transplanted to $15-\mathrm{cm}$ pots when the seedlings could be easily removed from the cell pack. After three weeks, seedlings with similar maturity and no 
sign of disease were then transplanted to 8.7-L pots to grow to full maturity. The greenhouse was normally under natural radiance with the capacity for high-pressure sodium (600 W HS200 deep reflector; Hortilux, Pijnacker, The Netherlands) supplemental lighting if the light intensity was below $50 \mathrm{~W} / \mathrm{m}^{2}$. The temperature and supplemental lighting regime in the greenhouse were set at $25 / 18^{\circ} \mathrm{C}$ and $14 / 10 \mathrm{~h}$ day/night.

Forty untreated broccoli heads were harvested eight weeks after transplanting. Meanwhile, another 40 plants were treated with $250 \mu \mathrm{M}$ MeJA in 0.1\% aqueous Triton X-100 (Sigma-Aldrich, St. Louis, MO, USA) solution three days before harvesting. The harvested broccoli heads were stored at $4{ }^{\circ} \mathrm{C}$ prior to cooking.

\subsection{Broccoli Preparation and Cooking Process}

\subsubsection{Broccoli Sample Preparation}

The harvested broccoli heads were chopped into 3-4-cm wide florets. After chopping, broccoli branchlets were randomized and were weighed $100 \mathrm{~g}$ for each replicate. Three replicates were used to represent the result from each cooking treatment. Broccoli was cooked for $2 \mathrm{~min}$ and $5 \mathrm{~min}$ using each cooking method to evaluate metabolite loss as a function of cooking time. For all three cooking methods, 2-min treatment and 5-min treatment were conducted separately, so no interruption occurred in 5-min treatment.

\subsubsection{Boiling Treatment}

For boiling treatment, the broccoli florets were immersed in $700 \mathrm{~mL}$ boiling water for 2 or $5 \mathrm{~min}$, following which they were immediately removed from the boiling water and transferred to an ice-water bath.

\subsubsection{Steaming Treatment}

For steaming treatment, the broccoli florets were evenly spread out without any overlap on a stainless-steel steamer basket, which was placed over $700 \mathrm{~mL}$ boiling water for 2 and 5 mins. Steamed broccoli was immediately transferred to an ice-water bath for rapid cooling.

\subsubsection{Microwaving Treatment}

For microwaving treatment, a microwave oven (Whirlpool Corporation, Benton Harbor, MI 49022, USA) was used at $50 \%$ power (maximum power: $1000 \mathrm{~W}$ ). The broccoli florets were placed on a plate and covered with a paper towel soaked in $50 \mathrm{~mL}$ water. The broccoli samples were microwaved for 2 or $5 \mathrm{~min}$ and were then transferred to an ice water bath for cooling. All cooked broccoli samples were packed in a zipper bag, which were then placed in a 68-L container (Sterilite, Townsend, MA 01469, USA) and moved to a $-20{ }^{\circ} \mathrm{C}$ walk-in cooler prior to lyophilization.

\subsubsection{Cooking Water Volume Adjustjment for Quantification}

The volume of the cooking water of the boiling and steaming treatments was adjusted to $700 \mathrm{~mL}$ before collecting in $50 \mathrm{~mL}$ twist tubes. For the microwaved sample, the damp paper towel was first rinsed in $200 \mathrm{~mL}$ water, after which the water was manually squeezed out of the paper towel and the volume was adjusted to $700 \mathrm{~mL}$. All cooking water samples were stored in a $4{ }^{\circ} \mathrm{C}$ walk-in cooler for further analysis.

\subsection{Quantification of Glucosinolates (GSLs) via UHPLC-DAD}

GSLs in samples were analyzed using a previously published method [28] with slight modifications. A quantity of $75 \mathrm{mg}$ of freeze-dried powdered material was weighted in a 2-mL screw top minicentrifuge tube. The samples were then extracted with $0.75 \mathrm{~mL} 70 \%$ aqueous methanol and placed onto a $95{ }^{\circ} \mathrm{C}$ shaking heating block for $10 \mathrm{~min}$. After $10 \mathrm{~min}$ incubation, samples were cooled down on the ice 
for $5 \mathrm{~min}$ and $187.5 \mu \mathrm{L}$ internal standard ( $0.907 \mathrm{mM}$ glucosinalbin, isolated from Sinapis alba) was added to each sample. The samples were vortexed for $10 \mathrm{~s}$ and then centrifuged at $12,000 \times g$ for $10 \mathrm{~min}$. The supernatant was collected in a new 2-mL microtube and stored on ice. The pellet was then re-extracted using the same condition described above, and the supernatant was collected into the corresponding 2-mL microtube from the last extraction. The mixed supernatant was then vortexed for $10 \mathrm{~s}$. The proteins in the supernatant were precipitated with $0.15 \mathrm{~mL}$ of a 1:1 mixture of $1 \mathrm{M}$ lead acetate and $1 \mathrm{M}$ barium acetate and centrifuged at $12,000 \times \mathrm{g}$ for $3 \mathrm{~min}$. DEAE Sephadex A-25 resin (GE Healthcare, Piscataway, NJ, USA) was prepared in a ratio of $20 \mathrm{~g}$ resin to $100 \mathrm{~mL}$ deionized water before the extraction, and $1 \mathrm{~mL}$ of well-mixed resin was added into a Poly-Prep column before the desulfation of GSL. After the resin was drained, the supernatant was poured into the Poly-Prep column. The resin was then washed with $3 \mathrm{~mL} 0.02 \mathrm{M}$ pyridine acetate and then $3 \mathrm{~mL}$ deionized water. Once the water eluted out of the column, $500 \mu \mathrm{L} 20 \mathrm{U} / \mathrm{mL}$ sulfatase solution was prepared from crude Helix pomatia type-1 arylsulfatase powder (Sigma-Aldrich, St. Louis, MO, USA) and incubated for desulfation of GSL overnight at room temperature. After overnight incubation, desulfo-GSL was eluted into 2-mL microtubes with $1.5 \mathrm{~mL}$ deionized distilled water, and a syringe with a $0.2-\mu \mathrm{m}$ nylon filter was used to filter the extract and move the extract into chromatography vials.

The separation and detection of GSLs was conducted on a Nexera-i LC 2040C ultra-high-performance liquid chromatography (UHPLC) (Shimadzu, Kyoto, Japan) machine coupled with a photo diode array detector (DAD). The DAD was set to monitor the absorbance at $229 \mathrm{~nm}$. The filtered samples were injected into the Kromasil reverse-phase C18 HPLC column $(1.8 \mu \mathrm{m}$, $100 \mathrm{~mm} \times 2.1 \mathrm{~mm}$ i.d., $100 \AA$ ) at $40^{\circ} \mathrm{C}$. The selection of mobile phase was deionized distilled water for solvent $\mathrm{A}$ and $100 \%$ acetonitrile for solvent $\mathrm{B}$ under the flow rate of $1 \mathrm{~mL}$ per minute, and gradient elution was conducted: from $0 \% \mathrm{~B}$ at $0 \mathrm{~min}$ to $4 \% \mathrm{~B}$ at $7 \mathrm{~min}, 20 \% \mathrm{~B}$ at $20 \mathrm{~min}, 25 \% \mathrm{~B}$ at $35 \mathrm{~min}, 80 \% \mathrm{~B}$ at $36 \mathrm{~min}$, then $80 \% \mathrm{~B}$ at $40 \mathrm{~min}$. The system was set to $0 \% \mathrm{~B}$ at $41 \mathrm{~min}$ until $50 \mathrm{~min}$ to reset the column condition for the next injection. Relative response factor (RRF) was adapted for quantification and the quantification was based on the internal standard, glucosinalbin, of which the RRF was 0.5 [29]. For other detected GSLs, the determined RRF values of glucoiberin, progoitrin, glucoraphanin, sinigrin, gluconapin 1.11, glucoerucin 1.00, glucobrassicin 0.29 , 4-methoxyglucobrassicin 0.25 , gluconasturtiin neoglucobrassicin, 1-hydroxyglucobrassicin/4-hydroxyglucobrassicin were 1.07, 1.09, 1.07, 1, 1.11, 1.00, $0.29,0.25,0.95,0.20$ and 0.28, respectively [29-31]. The presence of 1-hydroxyglucobrassicin in Brassica plants was rarely reported [30,32], but we do not want to exclude the possibility of 1-hroxyglucobrassicin in the sample because we detected significantly higher transcript abundance of CYP781F4 under the same treatment condition in 'Red Russian' kale using the primers designed with the published sequence information of the Brassica oleracea database [33].

To tentatively identify the desulfo-GSL present in the sample, a LC-tandem mass spectrometry (MS) system Waters 32 Q-Tof Ultima spectrometer coupled to a Thermo Accela 1200 UHPLC system coupled to a heated ESI source and to a Q Exactive high-resolution (HR) quadrupole and orbitrap LC-MS/MS (Thermo Scientific, Waltham, MA, USA) was employed. Identification information was obtained by comparing the previous publication from our lab [30], the fragmentation diagnostic ions presented in Kusznierewicz's work [34], and the information from Clarke's previous work [29].

GSLs in cooking water was determined using the same protocol mentioned above with slight modification. Precipitation of protein in GSL cooking water was completed with the addition of $0.37 \mathrm{~mL}$ of a 1:1 $1 \mathrm{M}$ barium acetate: $1 \mathrm{M}$ acetate acetate mixture and followed the same steps described in the last section.

\subsection{Quantification of GSL Hydrolysis Products via GC-MS}

$50 \mathrm{mg}$ freeze-dried broccoli powder was weighed in a 2-mL Teflon tube (Fisher Scientific, Waltham, MA, USA) and $1 \mathrm{~mL}$ distilled water was added for GSL hydrolysis. Tubes were stored inside a drawer to prevent light exposure. Under this setting, GSL hydrolysis products were generated by endogenous myrosinase at room temperature for $24 \mathrm{~h}$. To capture GSL hydrolysis products, $1 \mathrm{~mL}$ 
dichloromethane was added after a $24 \mathrm{~h}$ reaction, and the samples were vortexed and centrifuged after $2 \mathrm{~min}$ at $12,000 \times g$ to separate the liquid into aqueous and organic layers. After the centrifuge, the lower layer (dichloromethane) was pipetted out into a $1.5-\mathrm{mL}$ vial and analyzed using Trace 1310 gas chromatography (GC) (Thermo Fisher Scientific, Waltham, MA, USA) with a single quadrupole MS detector system (ISQ QD, Thermo Fisher Scientific, Waltham, MA, USA) and an autosampler (Triplus RSH, Thermo Fisher Scientific, Waltham, MA, USA). Samples were injected into a 30-m Rxi-5Sil MS capillary column $(0.25 \mathrm{~mm}, 0.25 \mathrm{~m}, \mathrm{w} / 10 \mathrm{~m}$ Integra-Guard Column; Restek, Bellefonte, PA, USA) for analyte separation. The temperature of the injector was set at $270{ }^{\circ} \mathrm{C}$ and the temperature of the detector was $275^{\circ} \mathrm{C}$. The GC temperature programming was set as below: initial temperature $\left(40^{\circ} \mathrm{C}\right)$ was held for $2 \mathrm{~min}$ and the oven temperature was increased to $320^{\circ} \mathrm{C}$ at a $15^{\circ} \mathrm{C} \mathrm{min}-1$ rate. When the oven reached $320^{\circ} \mathrm{C}$, this temperature was held for another $4 \mathrm{~min}$ to slightly bake the column to wash out remaining analytes and prepare for the next injection. The flow rate of the carrier gas, helium, was set at $1.2 \mathrm{~mL}$ per minute. Identification of detected MS fragments was based on the information the National Institute of Standards and Technology (NIST) library or previous publications [35].

\subsection{Quantification of Myrosinase Activity and Nitrile Formation via GC-MS}

Myrosinase activity and nitrile formation were measured to estimate the interaction between epithiospecifier protein (ESP) levels and epithiospecifier modifier 1 (ESM1) based on published methods. Myrosinase activity was calculated as the total hydrolysis product hydrolyzed from GSLs within $60 \mathrm{~min}$ of incubation. One unit is defined as $1 \mu \mathrm{mol}$ total GSL hydrolysis product released per minute. Nitrile formation was conducted by incubating crude protein extracts from broccoli samples with concentrated horseradish root extract. Horseradish extract was utilized in this analysis because it served as a highly saturated exogenous substrate source of sinigrin and gluconasturtiin GSL profile of horseradish, and it can minimize the reaction of endogenous myrosinase in broccoli with endogenous GSL substrates [36,37].

A quantity of $75 \mathrm{mg}$ freeze-dried broccoli powder was weighed in a 2-mL microcentrifuge tube and then added to $1.5 \mathrm{~mL}$ concentrated horseradish root extract. The samples were vortexed for $10 \mathrm{~s}$ and then centrifuged at $12,000 \times \mathrm{g}$ for $5 \mathrm{~min}$. A quantity of $0.5 \mathrm{~mL}$ of the supernatant was transferred to 1.5-mL Teflon centrifuge tubes (Savillex Corporation, Eden Prairie, MN, USA) with the addition of $0.5 \mathrm{~mL}$ before the incubation. To prevent the samples from potential light exposure, incubation was conducted in the drawer for $10 \mathrm{~min}$ at room temperature. After the incubation, samples were vortexed and centrifuged at $12,000 \times g$ for $4 \mathrm{~min}$ and the organic layer (dichloromethane) was collected into chromatography vials. Samples were then analyzed by the GC-MS system with the same thermal programming (in Section 2.4) for GSL hydrolysis product profiling. The calibration curves of allyl isothiocyanate, 2-phenthyl isothiocyanate, and 3- phenylpropionitrile (Sigma-Aldrich, St. Louis, MO, USA) were used for quantification. The calibration curve of allyl isothiocyanate was also applied to quantify 1-cyano-2,3-epithiopropane.

\subsection{Measurement of Electrical Conductivity in Cooking Water}

Electrical conductivity (EC) was measured using TechPro II $^{\mathrm{TM}}$ (Myron $\mathrm{L}^{\circledR}$, Carlsbad, CA, USA). The probe was calibrated first with deionized water. Deionized water was also used to rinse out the sampling probe thrice between measurements and the sampling probe was dried using Kim wipes before the measurement. EC was measured thrice for each cooking water sample and the averaged value of each sample was used for statistical analysis.

\subsection{Untargeted Primary Metabolites by GC-MS}

GC-MS, as described in Section 2.4, was also used to determine the primary metabolite profile in broccoli samples with derivatization. Primary metabolites were extracted using a previously published study [38] with slight modifications. Aquantity of $50 \mathrm{mg}$ of broccoli lyophilized powder was weighed in 2-mL microcentrifuge tubes and $1.4 \mathrm{~mL}$ methanol was then added into the samples for extraction. 
A quantity of $80 \mu \mathrm{L}$ of internal standard $(10 \mathrm{mg} / \mathrm{mL}$ ribitol) was also added into the samples. After $10 \mathrm{~s}$ of vertexing, the extracts were centrifuged and the supernatants were poured into a new set of 2-mL micro tubes. Quantities of $375 \mu \mathrm{L}$ of $-20{ }^{\circ} \mathrm{C}$ chloroform and $700 \mu \mathrm{L} 4{ }^{\circ} \mathrm{C}$ water were added into samples for polar compound fractionation. The extracts were then vortexed for $20 \mathrm{~s}$, then centrifuged and transferred to $1.5-\mathrm{mL}$ tubes for drying. The drying procedure was completed in a Vacufuge $\mathrm{T}^{\mathrm{TM}}$ concentrator (Eppendorf, Thermo Fisher Scientific, Waltham, MA, USA). After drying, samples were derivatized by incubating the extract with $50 \mu \mathrm{L}$ of $40 \mathrm{mg} / \mathrm{mL}$ methoxyamine hydrochloride in pyridine for $90 \mathrm{~min}$ on a $37^{\circ} \mathrm{C}$ heating block. After this incubation, $70 \mu \mathrm{L} N$-Methyl- $N$-(trimethylsilyl) trifluoroacetamide (MSTFA) with $1 \%$ trimethylchlorosilane (TMCS) was added into the sample for another 30-min incubation on a $37^{\circ} \mathrm{C}$ heating block. Extracted analytes were then injected into the same GC-MS system mentioned in Section 2.4 for profiling. The MS detection used positive electron impact mode (EI) with a $\mathrm{m} / z$ 40-500 scan range and ionization energy at $70.0 \mathrm{eV}$.

After the GC-MS analyses were completed, raw files generated from primary metabolite profiling were converted to mzXML file format by RawConverter [39]. The detection and alignment of extracted peaks were completed using the XCMS package in R studio with default settings [40]. In R multivariate or univariate statistics by MetaboAnalyst [40], data underwent Pareto scaling and was normalized to a unique ion peak $(319 \mathrm{~m} / \mathrm{z})$ generated from the spiked internal standard (ribitol). Tentative metabolite annotation was done depending on the fragment pattern and retention times generated by authentic standards using the same chromatogram conditions or comparing with the mass spectra present in the NIST library.

\subsection{Univariate and Multivariate Analyses}

JMP 14 (SAS Institute, Cary, NC, USA) was used for the statistical analyses. Two-way univariate analysis of variance (two-way ANOVA) was used to determine the effect of MeJA treatment and cooking method on the metabolites. Data was separated into (A) raw and 2-min cooking and (B) raw and 5-min cooking when two-way ANOVA was used. A slice test was used to determine the effect of MeJA on metabolites when the interaction between two factors (MeJA treatment and cooking method) was significant at $p \leq 0.05$. If no interaction was detected, a Student's T-test was used to determine the effect of MeJA on metabolites under the same cooking method, and the significance was $p \leq 0.05$.

For primary metabolite analysis, the acquired chromatograms, converted to $\mathrm{mzXML}$, were used to conduct partial least-square discrimination analysis, variable importance in projection value estimation, and ANOVA in MetaboAnalyst [40].

\section{Results and Discussion}

\subsection{Effect of MeJA and Cooking Method on Glucosinolate Profile}

A total of 10 GSLs were detected in broccoli samples, and the results of two-way ANOVA (Table S1) showed a significant interaction $(p \leq 0.05)$ between MeJA treatment and cooking method in total indole GSLs and total GSL, irrespective of the cooking time. The concentration of total GSL in MeJA-treated broccoli was significantly higher $(p \leq 0.001)$ in MeJA-treated broccoli among all cooking methods (Figure 1). The concentration of total GSL in raw, steamed and microwaved MeJA-treated broccoli was the greatest across all samples by post-hoc Tukey's HSD test at $p \leq 0.05$, with no significant difference between each other. Boiling led to the maximum loss of GSL among all MeJA-treated broccoli, which corresponded well with previous studies $[10,11]$. However, the concentration of glucoraphanin was significantly reduced $(p \leq 0.05)$, which was different from a previous study [6] that found that the concentration of glucoraphanin did not differ between control and $500 \mu \mathrm{M}$ MeJA-treated broccoli [6]. The decrease may occur during postharvest, since glucoraphanin was rapidly reduced in MeJA-treated broccoli 10 days after the harvest when stored at $4{ }^{\circ} \mathrm{C}$ [6]. 


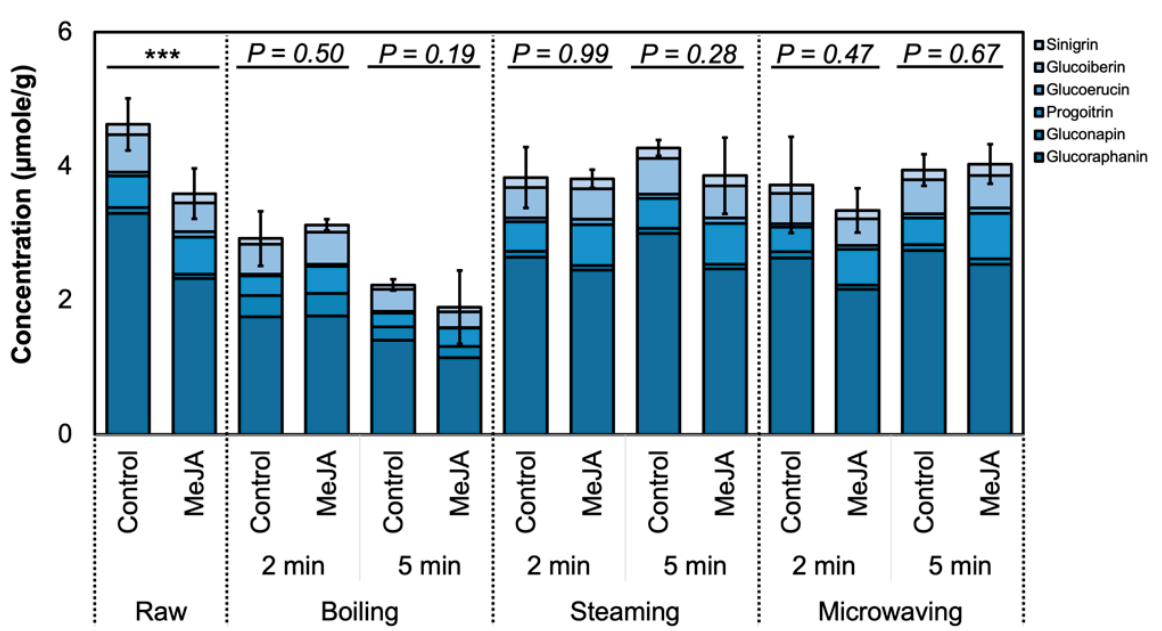

A
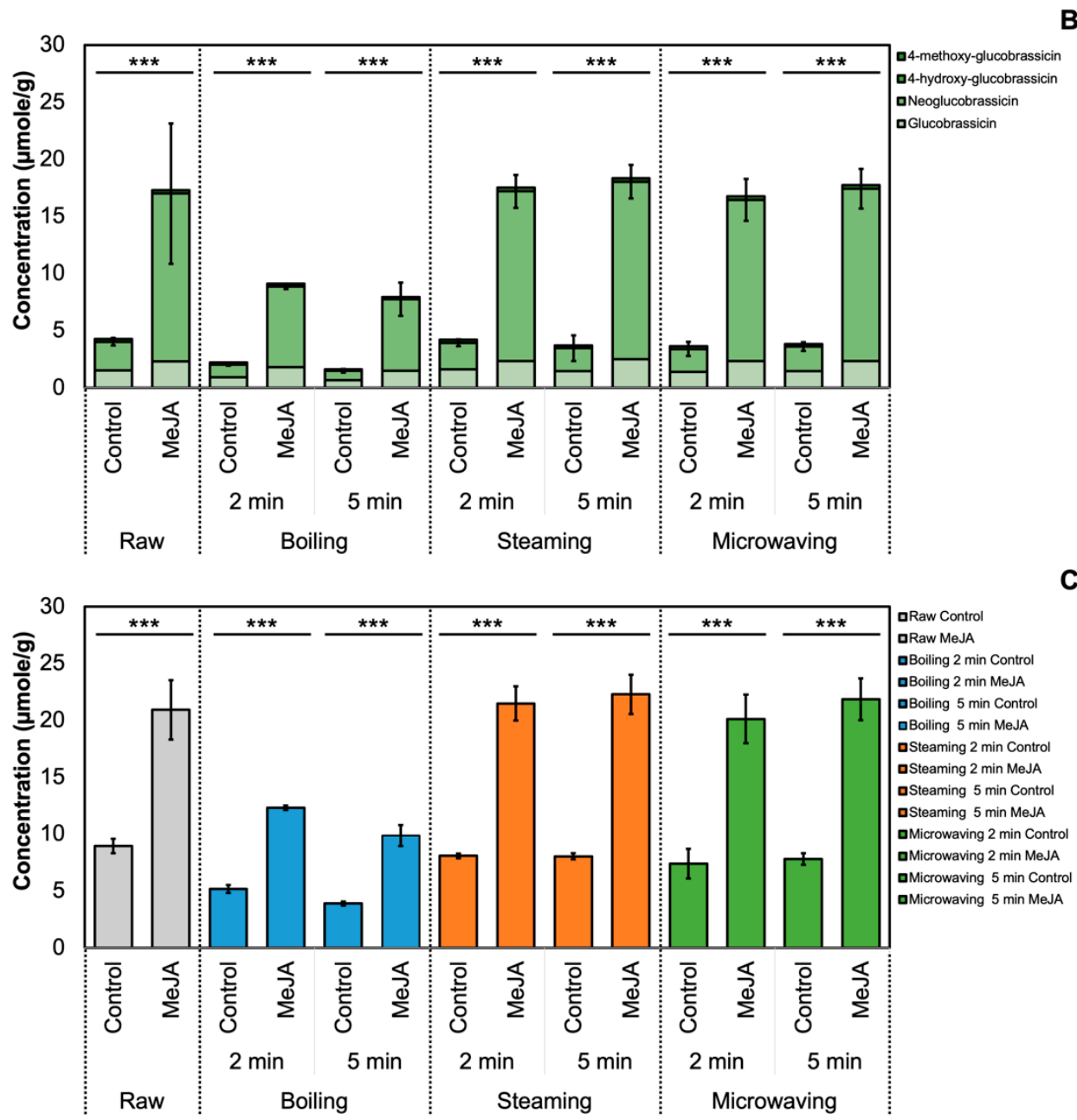

Cooking treatment

Figure 1. Effect of cooking and $250 \mu \mathrm{M}$ MeJA treatment on total aliphatic glucosinolates (A), total indole glucosinolates (B), and total glucosinolates (C) in 'Green Magic' broccoli. C: control broccoli; M, MeJA-treated broccoli. Asterisk $\left.{ }^{*}\right)=$ detected significant different by Student's T-test $(p \leq 0.05, n=3)$ with a significant interaction between MeJA treatment and cooking treatment detected (Supplementary Table S2). ${ }^{* * *}, p \leq 0.001$. 
Regardless of different cooking methods and durations, the total GSL amount in MeJA-treated broccoli was still higher than in the non-treated broccoli. This suggests that the increased GSL concentration in broccoli samples was solely affected by MeJA treatment, and the effect of MeJA was not affected by cooking methods. Reports show that the change in GSL during the cooking process can depend on GSL structure [41], plant matrix [42], or cellular environment [43]. The use of larger volumes of cooking water (boiling) also leads to a higher loss of GSL [44]. GSL loss during the cooking process may be attributed to cell lysis and thermal degradation [41]. GSL concentration in the cooking water was measured and varied between cooking methods. The greatest concentration of total GSL in cooking water was detected in 2-min boiling water from MeJA-treated broccoli (Table S1) and no interaction of MeJA treatment and cooking method was detected. Results were consistent with previous literature where boiling was reported to lead to a great loss of GSL [41,45]. Electrical conductivity (EC) of the cooking water was also measured (Table S4; Figure 2) but interactions from the two factors (MeJA treatment and cooking methods) were not detected ( $p=0.48$ for 2-min cooking of broccoli and $p=0.12$ for 5-min cooking of broccoli). In 5-min boiling and steaming cooking treatment, the EC value of the cooking water from MeJA-treated broccoli is significantly lower than that from the control broccoli. From the two-way ANOVA analysis for the 5-min cooking experiment, the effect of MeJA treatment on the EC value was significant, while there was no significant effect on EC in cooking water from 2-min cooking (Table S3). The EC value of the boiling water of MeJA-treated and untreated broccoli was also the highest among all the samples, which suggested a high level of cell lysis [10] and leaching of GSL into the cooking water.

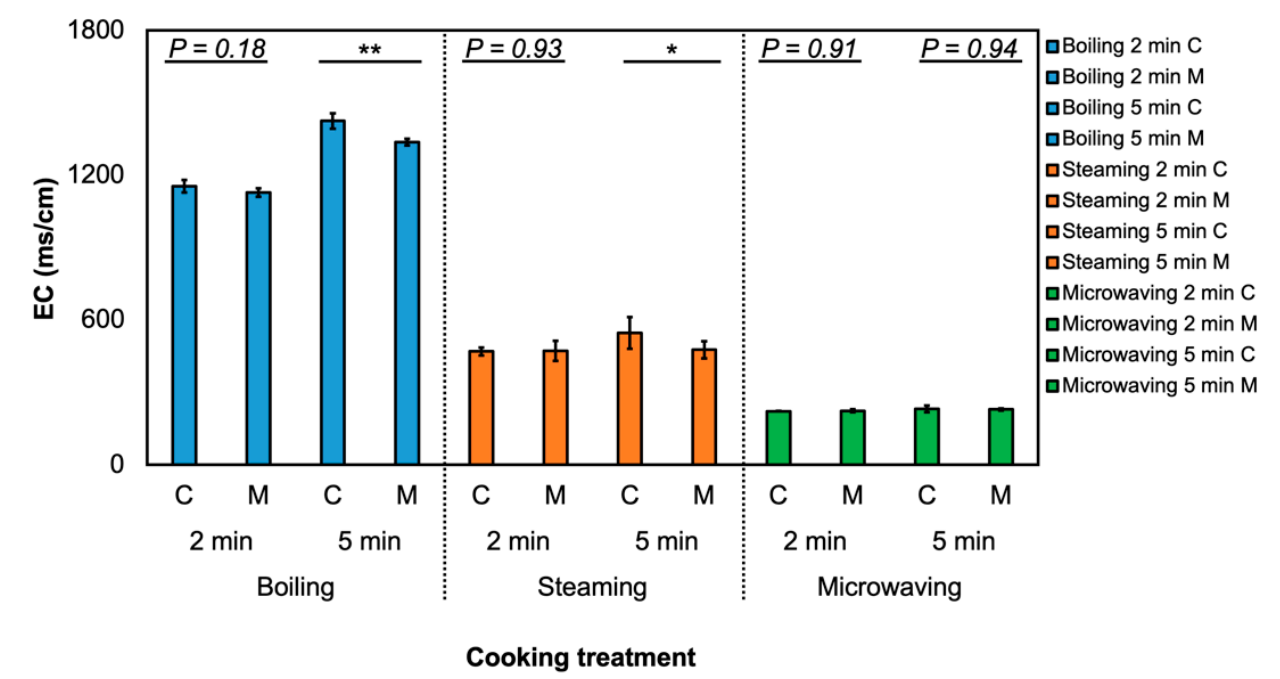

Figure 2. Electrical conductivity in cooking water. Asterisk $\left(^{*}\right)$ indicates significant difference with or without MeJA within the same cooking treatment by Student's T-test $(p \leq 0.05, n=3)^{\mathrm{ns}},{ }^{*}, p \leq 0.05$; **, $p \leq 0.01$. The first letter, $\mathrm{C}$ or $\mathrm{M}$, indicates the control or methyl jasmonate-treated group. The second letter, B, M, or S, indicates different cooking treatments including boiling, microwaving, and steaming. The last number after the $\mathrm{B}, \mathrm{M}$, or $\mathrm{S}$ indicates the duration of the cooking method (mins).

MeJA treatment might change the cellular environment [46] and alter cell structure by changing the cell wall composition [47-49], which affects leaching of GSL from the cell. In this study, total GSL content in MeJA-treated broccoli was 1.6-fold higher than in untreated control broccoli after 5-min boiling, which was considered the most disruptive cooking method in this study (Figure 1). Furthermore, total GSL amount in MeJA-treated broccoli was significantly higher than in all steamed samples and in the 5-min microwaving groups compared to the untreated broccoli. For milder cooking methods (2-min steaming or 2-min microwaving), the concentration of total GSL in MeJA-treated broccoli was 2.5-fold higher than in the untreated control broccoli. Many studies have suggested that intact GSL in food can be hydrolyzed via the myrosinase-like enzyme produced by the human gut microbiota [50], while the efficiency may depend on the individual and the types of microbes. Moreover, a 2020 study 
from Liou et al. has revealed the bacteria species that convert GSL into isothiocyanates in human gut [51].

Although health-promoting effects of indole GSL and its hydrolysis products are reported, it was also suggested that neoglucobrassicin may pose risks to humans. Neoglucobrassicin in pok choi (treated with $2 \mathrm{mM} \mathrm{MeJA}$ ) sprout juice was reported to have high mutagenic activity using human sulphotransferase (SULT) 1A1 (hSULT1A1) [24]. I3C, a hydrolysis product from glucobrassicin, was also reported to have Janus properties in animal models [25]. Considering that anticarcinogenic and carcinogenic effects of indole GSLs and hydrolysis products have both been reported previously, the GSL profile provided in this study may be important for dieticians and professionals once a better understanding is reached. If a certain concentration of neoglucobrassicin is determined to be health-promoting in the future, then MeJA treatment can be a valuable tool to deliver high GSL into the human digestive system via Brassica crops as a vehicle.

\subsection{Effect of MeJA Treatment and Cooking Method on Myrosinase Activity and GSL Hydrolysis Products in} 'Green Magic' Broccoli

In raw broccoli, $250 \mu \mathrm{M}$ MeJA treatment significantly increased myrosinase activity by $37 \%$ (Figure 3). We also indirectly measured epithiospecifier protein (ESP) activity by incubating broccoli crude protein and horseradish GSL extract together [36]. Nitrile formation (\%) of gluconasturiin was reduced, although the result was not statistically significant ( $p=0.06$, not presented).

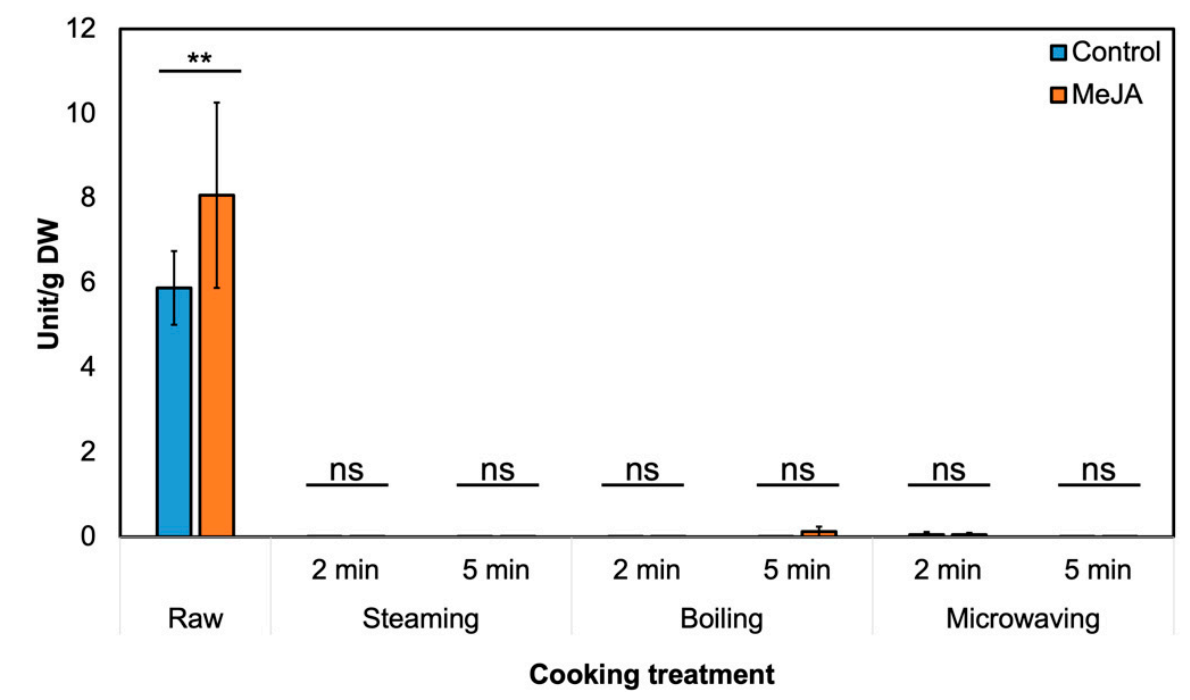

Figure 3. Effect of cooking and $250 \mu \mathrm{M}$ MeJA treatment on myrosinase activity in broccoli. Asterisk $\left({ }^{*}\right)=$ detected significant difference with MeJA treatment by Student's T-test at $p \leq 0.05$ $(n=3)$. One unit $=1 \mu$ mole of total released GSL hydrolysis products in 1 minute. ${ }^{\text {ns }}$, not significant; ${ }^{* *}, p \leq 0.01$.

Eleven GSL hydrolysis products were detected in raw broccoli, including ITC, nitriles, indoles and oxazolidine-thione (Table S5). With the substantial increase in neoglucobrassicin and glucobrassicin after MeJA treatment (Table S3), the levels of hydrolysis products derived from neoglucobrassicin (NMI3C, $\mathrm{N}$-methoxyindole-3-carboxyaldehyde (NMI3CA), $N$-methoxyindole-3-acetonitrile (NMI3ACN)), and indole-3-carbinol (I3C) from glucobrassicin were significantly higher $(p \leq 0.05)$ in raw MeJA-treated broccoli than in untreated broccoli. NMI3C and I3C were reported to have anti-inflammatory and chemopreventive effects [23,52]; moreover, NMI3C may be a stronger inhibitor than I3C of tumor development [53]. Therefore, the increase in the levels of these compounds after MeJA treatment might improve the nutritional value of raw broccoli. NMI3C and NMI3ACN levels were significantly higher in MeJA-treated broccoli for most of the cooking methods (Table S5). However, other GSL hydrolysis 
products were not affected by the MeJA treatment (Table S5); therefore, the effect of MeJA on hydrolysis products may vary with precursor GSL.

In Figure 4, the abundance of GSL hydrolysis products in cooked samples was lower compared to the raw samples. This result corresponds well with the myrosinase activity decrease in the cooked samples (Figure 3). As the abundance of GSL hydrolysis produces contributes significantly to broccoli's taste and determines consumer acceptance [54], it is plausible that cooking treatment of MeJA-treated broccoli may be more acceptable to average consumers, while these broccolis still contain a high amount of GSL before cooking.

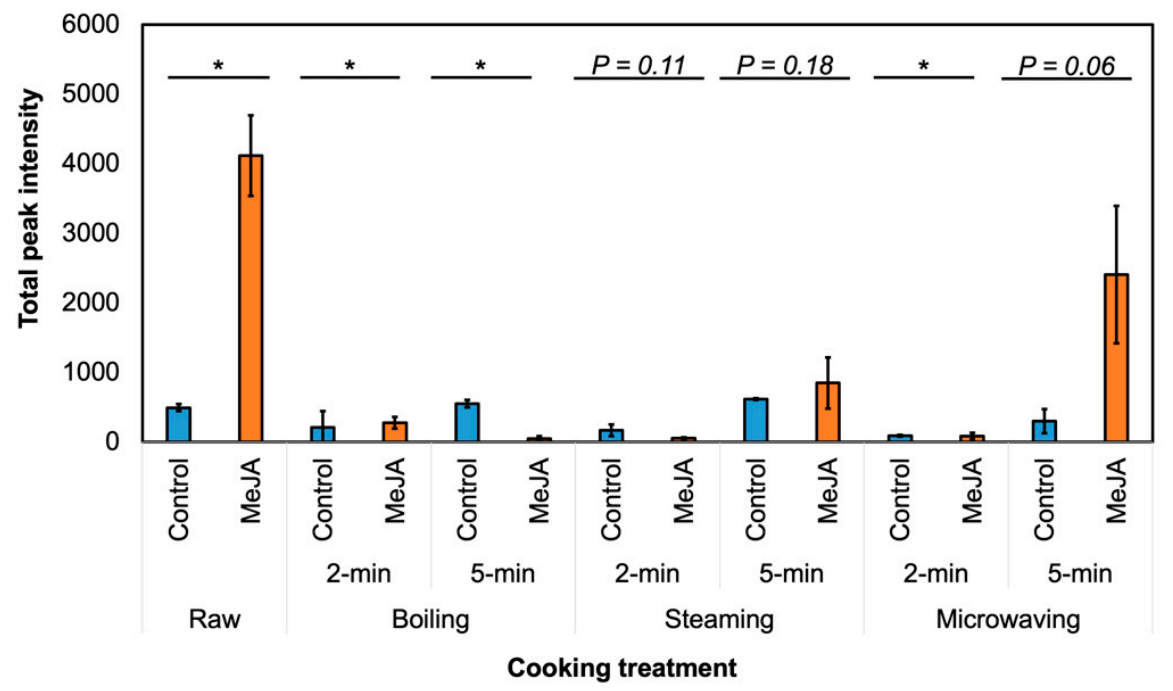

Figure 4. Total peak intensity of glucosinolate hydrolysis products in raw and cooked broccoli. MeJA, broccoli with $250 \mu \mathrm{M}$ methyl jasmonate treatment. Asterisk $\left(^{*}\right)=$ significant difference with or without MeJA by Student's T-test ( $p \leq 0.05, n=3)$ under the same cooking conditions. ${ }^{\text {ns }},{ }^{*}, p \leq 0.05$.

Myrosinase activity was close to zero in all the cooked samples (Figure 3). Myrosinase inactivation during the cooking process may reduce the content of all hydrolysis products, as no significant differences in hydrolysis product levels were observed between the majority of cooked MeJA-treated and untreated broccolis. Therefore, although MeJA treatment might increase myrosinase activity in the raw material, the effect was not observed after 2-min boiling, steaming, or microwaving [53]. We recently found that MeJA-treated raw broccoli significantly reduced consumer preference, but treatment did not significantly change consumer preference after cooking because of the low concentration of hydrolysis products from neoglucobrassicin by myrosinase inactivation [54]. Thus, MeJA-treated broccoli can be favorable delivered as frozen broccoli or cooked broccoli rather than raw. Previous results reported that there is very low or no myrosinase activity in frozen broccoli, which does not form sulforaphane [55], but it does not produce an off-flavor from neoglucobrassicin in this case.

\subsection{Effect of MeJA and Cooking Method on Primary Metabolites in 'Green Magic' Broccoli with or without MeJA Treatment}

Studies have shown that MeJA may change primary metabolites, including sugar, amino acids, and organic acids, by regulating the balance between growth and defense activities $[30,56]$. These primary metabolites can be valuable nutrition components; however, the effect of MeJA treatment on primary metabolites in cooked broccoli has not been studied. Partial least-square discrimination analysis (PLS-DA) (Figure 5) was used to identify potential biomarkers with variable importance in projection (VIP) values over 1.5, which indicates that this metabolite contributed greatly to the differences between groups (Table S6). 
A

B
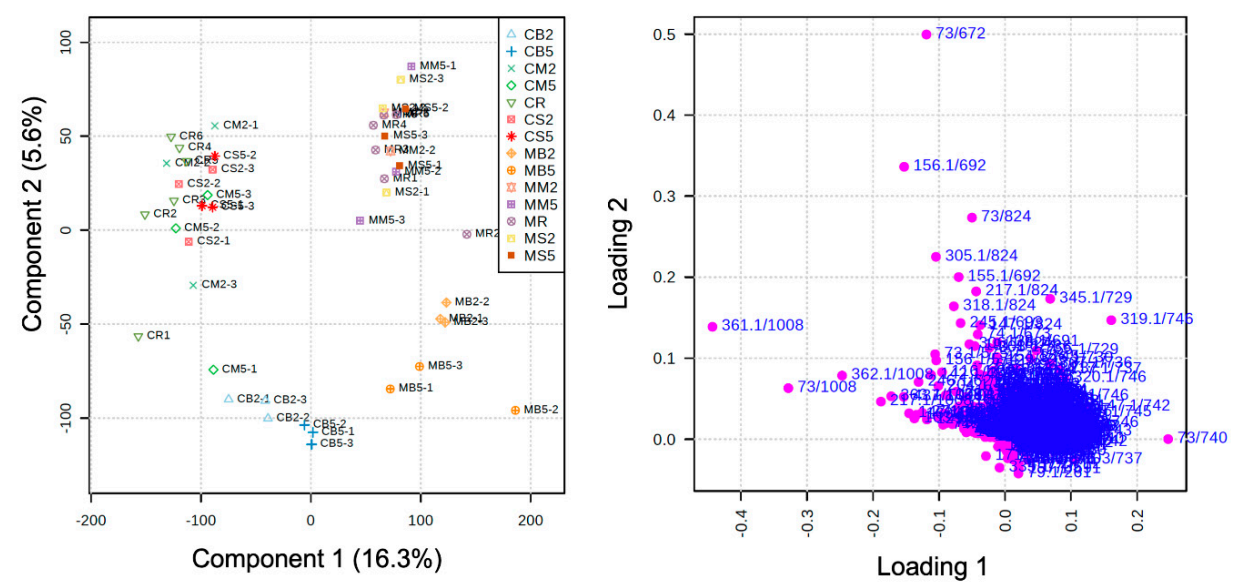

Figure 5. Partial least-square discrimination analysis score plot (A) and loading plot (B) derived from raw (control raw: CR; methyl jasmonate-treated raw: MR) and cooked control and methyl jasmonate-treated broccoli samples. The first letter, $\mathrm{C}$ or $\mathrm{M}$, indicates the control or methyl jasmonate-treated group. The second letter, $\mathrm{B}, \mathrm{M}$, or $\mathrm{S}$, indicates different cooking treatments including boiling, microwaving, and steaming. The last number after $\mathrm{B}, \mathrm{M}$, or $\mathrm{S}$ indicates the duration of the cooking method (mins).

In the raw broccoli, MeJA treatment significantly reduced $(p \leq 0.05)$ oxoproline, glutamic acid, myo-inositol, and sucrose content (Table 1). This was consistent with published studies where MeJA treatment reduced sugars and amino acids in other Brassica vegetables [35,57]. Therefore, MeJA-mediated reduction in sugar and amino acid content was common for many Brassica species. The VIP values of primary metabolites, including sucrose, glucose, fructose, myo-inositol, oxoproline, and quininic acid, were consistently high (>1.5) with all cooking methods; hence, changes in the levels of these compounds were due to the effect of MeJA and not because of cooking methods. In other words, $250 \mu \mathrm{M}$ MeJA treatment changed the amount of individual primary metabolites in raw broccoli, and this effect was still observed after all cooking methods. 
Table 1. Fold changes of primary metabolites in MeJA-treated 'Green Magic' compared to untreated broccoli under the same cooking treatment.

\begin{tabular}{|c|c|c|c|c|c|c|c|c|c|c|c|c|c|}
\hline \multirow{2}{*}{\multicolumn{2}{|c|}{ Cooking Method }} & \multicolumn{7}{|c|}{ Amino Acids $^{\dagger}$} & \multicolumn{4}{|c|}{ Sugar and Sugar Derivatives } & \multirow{2}{*}{$\begin{array}{c}\text { Organic Acids } \\
\text { Quinic Acid }\end{array}$} \\
\hline & & \multirow{2}{*}{$\begin{array}{c}\begin{array}{c}\text { Oxo- } \\
\text { Proline }\end{array} \\
0.58 *\end{array}$} & \multirow{2}{*}{$\begin{array}{c}\text { Glu } \\
0.42 *\end{array}$} & \multirow[t]{2}{*}{ Val } & \multirow[t]{2}{*}{ Pro } & \multirow[t]{2}{*}{ Ser } & \multirow[t]{2}{*}{ Ile } & Ala & Fructose & Glucose & Sucrose & myo-Inositol & \\
\hline Rav & & & & & & & & & 0.94 & 1.06 & $0.28 *$ & $0.91 *$ & 1.35 \\
\hline \multirow{2}{*}{ Boiling } & $2 \mathrm{~min}$ & $0.77^{*}$ & $0.35 *$ & & & & & & $0.96 *$ & $1.09 *$ & $0.26 *$ & $0.90 *$ & $1.35 *$ \\
\hline & $5 \mathrm{~min}$ & 0.95 & 0.43 * & & & & & & 0.80 & 1.09 & 0.37 * & 0.84 & 1.73 \\
\hline \multirow{2}{*}{ Steaming } & $2 \min$ & $0.71 *$ & 0.40 * & $0.49 *$ & $0.40 *$ & 0.39 * & $0.38 *$ & & & 1.05 & $0.32 *$ & $0.74 *$ & $1.72 *$ \\
\hline & $5 \mathrm{~min}$ & 0.70 * & 0.35 * & 0.43 * & 0.35 * & 0.40 * & $0.26 *$ & & & 0.90 * & $0.26 *$ & $0.59 *$ & 1.54 * \\
\hline \multirow{2}{*}{$\begin{array}{c}\text { Micro- } \\
\text { waving }\end{array}$} & $2 \mathrm{~min}$ & $0.46^{*}$ & $0.43 *$ & 0.48 & $0.37 *$ & $0.36^{*}$ & & $0.69 *$ & 0.98 & $1.05 *$ & $0.26 *$ & $0.76^{*}$ & $2.04 *$ \\
\hline & $5 \mathrm{~min}$ & $0.62 *$ & $0.57^{*}$ & 0.57 * & 0.57 * & 0.56 * & & 0.86 * & 1.31 * & $1.38^{*}$ & $0.49 *$ & 1.02 & $2.33 *$ \\
\hline
\end{tabular}

† Abbreviations of amino acids: (1) Glu = glutamic acid, (2) Val $=$ valine, (3) Pro = proline, (4) Ser $=$ serine, (5) Ile $=$ isoleucine, and (6) Ala $=$ alanine. Asterisk $(*)=$ detected significant difference with or without MeJA treatment by Student's T-test at $p \leq 0.05$ based on the peak intensity $(n=3)$. 
MeJA treatment significantly increased the amount of quinic acid in the 2-min boiling, 2- and 5-min steaming, and 2- and 5-min microwaving samples. The levels of amino acids (oxoproline, glutamic acid, valine, isoleucine, proline, serine, and alanine) were significantly reduced by MeJA treatment, and those of glutamic acid, proline, and serine were reduced by $>50 \%$ (Table 1 ). The amounts of sucrose and myo-inositol were significantly lower in all MeJA-treated cooked broccoli; however, the amounts of glucose and fructose showed opposite trends. MeJA-treated broccoli contained significantly higher amounts of glucose after 2 min boiling and microwaving. In addition, the amount of fructose was significantly higher after 5 min microwaving of MeJA-treated broccoli.

It has been reported that amino acids can taste sweet, sour, bitter, or umami [58]. For example, glutamate is sometimes used as an umami ingredient in culinary practices. In this study, amino acids associated with bitterness (valine and isoleucine), sweetness (alanine, proline, and serine), and umami (glutamic acid) were all significantly changed by $250 \mu \mathrm{M}$ MeJA treatment. Sugars, such as sucrose, are associated with masking the bitterness of Brassica vegetables [59], and glucose correlates highly with the perception of sweetness [59]. In fact, $250 \mu \mathrm{M}$ MeJA treatment on 'Green Magic' broccoli was found to change the sensorial perception of raw broccoli but not of cooked broccoli, as determined by a consumer panel. The perceptible changes were mainly attributed to neoglucobrassicin and its hydrolysis products, not to amino acids or sugars [54]. Understanding the changes of primary metabolites in response to MeJA treatment and to various cooking methods may provide valuable insights regarding the nutritional and sensorial values of broccoli.

\section{Conclusions}

In this study, broccoli treated with $250 \mu \mathrm{M}$ MeJA contained significantly greater concentrations of GSL than untreated broccoli even after cooking by boiling, steaming or microwaving. Exogenous $250 \mu \mathrm{M}$ MeJA application will increase the nutritional value of cooked broccoli because of the increased concentration of GSL, without producing bad flavors from GSL hydrolysis products even after cooking. MeJA-treated broccoli has potential for use as a value-added ingredient and, since MeJA can be applied to other Brassica vegetables, this treatment may be used to enhance the nutritional value of other commodities once the health-promoting effects of indole GSL and the hydrolysis products are better understood.

Supplementary Materials: The following are available online at http://www.mdpi.com/2304-8158/9/6/758/s1. Table S1: Total glucosinolate profile ( $\mu$ mole $\cdot \mathrm{mL}$ ) in the cooking water collected from cooked broccoli with or without $250 \mu \mathrm{M}$ methyl jasmonate application (average $\pm \mathrm{SD}, n=3$ ), Table S2: Results of two-way ANOVA (MeJA treatment $\times$ cooking method) for glucosinolate profiles of 2-minute cooking (A) and of 5-min cooking (B), Table S3: Glucosinolate profile ( $\mu$ mole $\mathrm{g}^{-1}$ dry weight) of aliphatic glucosinolates (A) and indole and benzenic glucosinolates (B) in raw and cooked broccoli with or without $250 \mu \mathrm{M}$ methyl jasmonate application (average $\pm \mathrm{SD}, n=3$ ), Table S4: Two-way analysis of variance (ANOVA) of the electrical conductivity in cooking water of broccoli with or without $250 \mu \mathrm{M}$ MeJA treatment, Table S5: The peak intensity $(\times 103)$ of glucosinolate hydrolysis products in (A) raw and 2-min cooked and (B) raw and 5-min cooked "Green Magic" broccoli with or without $250 \mu \mathrm{M}$ methyl jasmonate application (average $\pm \mathrm{SD}, n=3$ ). Compound identification was based on a previous publication (Kim et al., 2017) or by comparison with data in the National Institute of Standards and Technology (NIST) library, Table S6: Primary metabolites that are high in variable influence on projection (VIP) by partial least-square discrimination analysis (PLS-DA) from raw and cooked 'Green Magic' broccoli with or without $250 \mu \mathrm{M}$ MeJA treatment by GC-MS analysis. All listed metabolites were significantly changed in ANOVA at $p \leq 0.05$.

Author Contributions: Experimental design, K.-M.K. and Y.-C.C.; conducting experiment and data analysis, Y.-C.C.; writing-original draft preparation, Y.-C.C.; writing—review and editing, K.-M.K., K.M., Y.-C.C. All authors have read and agreed to the published version of the manuscript.

Funding: This study is based on research that is supported by the National Institute of Food and Agriculture, U.S. Department of Agriculture, Hatch project number \#WVA 00722 and 112506.

Acknowledgments: We thank Matak for sharing the food science kitchen and Mengpei Liu, Lihua Zhang, Moo Jum Kim and Tylor Simpson for helping with the cooking experiment.

Conflicts of Interest: The authors declare no conflict of interest with regard to this work. 


\section{References}

1. Ambrosone, C.B.; McCann, S.E.; Freudenheim, J.L.; Marshall, J.R.; Zhang, Y.; Shields, P.G. Breast Cancer Risk in Premenopausal Women Is Inversely Associated with Consumption of Broccoli, a Source of Isothiocyanates, but Is Not Modified by GST Genotype. J. Nutr. 2004, 134, 1134-1138. [CrossRef] [PubMed]

2. Chen, Y.-J.; Wallig, M.A.; Jeffery, E.H. Dietary Broccoli Lessens Development of Fatty Liver and Liver Cancer in Mice Given Diethylnitrosamine and Fed a Western or Control Diet. J. Nutr. 2016, 146, 542-550. [CrossRef] [PubMed]

3. Baek, S.-A.; Jung, Y.-H.; Lim, S.-H.; Park, S.U.; Kim, J.K. Metabolic Profiling in Chinese Cabbage (Brassica rapa L. subsp. pekinensis) Cultivars Reveals that Glucosinolate Content Is Correlated with Carotenoid Content. J. Agric. Food Chem. 2016, 64, 4426-4434. [CrossRef] [PubMed]

4. Klopsch, R.; Witzel, K.; Artemyeva, A.; Ruppel, S.; Hanschen, F.S. Genotypic Variation of Glucosinolates and Their Breakdown Products in Leaves of Brassica rapa. J. Agric. Food Chem. 2018, 66, 5481-5490. [CrossRef] [PubMed]

5. Halkier, B.A.; Gershenzon, J. Biology and Biochemistry of Glucosinolates. Annu. Rev. Plant Biol. 2006, 57, 303-333. [CrossRef]

6. Ku, K.M.; Choi, J.H.; Kim, H.S.; Kushad, M.M.; Jeffery, E.H.; Juvik, J.A. Methyl Jasmonate and 1-Methylcyclopropene Treatment Effects on Quinone Reductase Inducing Activity and Post-Harvest Quality of Broccoli. PLoS ONE 2013, 8, e77127. [CrossRef]

7. Hu, R.; Xu, C.; Shen, G.; Jain, M.R.; Khor, T.O.; Gopalkrishnan, A.; Lin, W.; Reddy, B.; Chan, J.Y.; Kong, A.-N.T. Gene expression profiles induced by cancer chemopreventive isothiocyanate sulforaphane in the liver of C57BL/6J mice and C57BL/6J/Nrf2 (-/-) mice. Cancer Lett. 2006, 243, 170-192. [CrossRef]

8. Brown, A.F.; Yousef, G.G.; Jeffery, E.H.; Klein, B.P.; Wallig, M.A.; Kushad, M.M.; Juvik, J.A. Glucosinolate Profiles in Broccoli: Variation in Levels and Implications in Breeding for Cancer Chemoprotection. J. Am. Soc. Hortic. Sci. 2002, 127, 807-813. [CrossRef]

9. Vallejo, F.; Tomás-Barberán, F.; García-Viguera, C. Glucosinolates and vitamin C content in edible parts of broccoli florets after domestic cooking. Eur. Food Res. Technol. 2002, 215, 310-316. [CrossRef]

10. Jones, R.B.; Frisina, C.L.; Winkler, S.; Imsic, M.; Tomkins, R.B. Cooking method significantly effects glucosinolate content and sulforaphane production in broccoli florets. Food Chem. 2010, 123, $237-242$. [CrossRef]

11. Kapusta-Duch, J.; Kusznierewicz, B.; Leszczyńska, T.; Borczak, B. Effect of cooking on the contents of glucosinolates and their degradation products in selected Brassica vegetables. J. Funct. Foods 2016, 23, 412-422. [CrossRef]

12. Oliviero, T.; Verkerk, R.; Vermeulen, M.; Dekker, M. In vivo formation and bioavailability of isothiocyanates from glucosinolates in broccoli as affected by processing conditions. Mol. Nutr. Food Res. 2014, 58, 1447-1456. [CrossRef] [PubMed]

13. Ku, K.M.; Jeffery, E.H.; Juvik, J.A. Optimization of methyl jasmonate application to broccoli florets to enhance health-promoting phytochemical content: Meja enhancement of health-promoting phytochemicals in broccoli. J. Sci. Food Agric. 2014, 94, 2090-2096. [CrossRef] [PubMed]

14. Wu, J.; Wang, L.; Baldwin, I.T. Methyl jasmonate-elicited herbivore resistance: Does MeJA function as a signal without being hydrolyzed to JA? Planta 2008, 227, 1161-1168. [CrossRef] [PubMed]

15. Tamogami, S.; Noge, K.; Abe, M.; Agrawal, G.K.; Rakwal, R. Methyl jasmonate is transported to distal leaves via vascular process metabolizing itself into JA-Ile and triggering VOCs emission as defensive metabolites. Plant Signal. Behav. 2012, 7, 1378-1381. [CrossRef]

16. Kim, H.S.; Juvik, J.A. Effect of Selenium Fertilization and Methyl Jasmonate Treatment on Glucosinolate Accumulation in Broccoli Florets. J. Am. Soc. Hortic. Sci. 2011, 136, 239-246. [CrossRef]

17. Frerigmann, H.; Gigolashvili, T. MYB34, MYB51, and MYB122 Distinctly Regulate Indolic Glucosinolate Biosynthesis in Arabidopsis thaliana. Mol. Plant 2014, 7, 814-828. [CrossRef]

18. Thaler, J.S.; Stout, M.J.; Karban, R.; Duffey, S.S. Exogenous jasmonates simulate insect wounding in tomato plants (Lycopersicon esculentum) in the laboratory and field. J. Chem. Ecol. 1996, 22, 1767-1781. [CrossRef]

19. Campos, M.L.; Yoshida, Y.; Major, I.T.; de Ferreira, D.O.; Weraduwage, S.M.; Froehlich, J.E.; Johnson, B.F.; Kramer, D.M.; Jander, G.; Sharkey, T.D.; et al. Rewiring of jasmonate and phytochrome B signalling uncouples plant growth-defense tradeoffs. Nat. Commun. 2016, 7, 12570. [CrossRef] 
20. Becker, T.M.; Juvik, J.A. The Role of Glucosinolate Hydrolysis Products from Brassica Vegetable Consumption in Inducing Antioxidant Activity and Reducing Cancer Incidence. Diseases 2016, 4, 22. [CrossRef]

21. Jump, S.M.; Kung, J.; Staub, R.; Kinseth, M.A.; Cram, E.J.; Yudina, L.N.; Preobrazhenskaya, M.N.; Bjeldanes, L.F.; Firestone, G.L. N-Alkoxy derivatization of indole-3-carbinol increases the efficacy of the G1 cell cycle arrest and of I3C-specific regulation of cell cycle gene transcription and activity in human breast cancer cells. Biochem. Pharmacol. 2008, 75, 713-724. [CrossRef]

22. Sarkar, F.H.; Li, Y. Indole-3-Carbinol and Prostate Cancer. J. Nutr. 2004, 134, 3493S-3498S. [CrossRef]

23. Neave, A.S.; Sarup, S.M.; Seidelin, M.; Duus, F.; Vang, O. Characterization of the N-methoxyindole-3-carbinol (NI3C)-Induced Cell Cycle Arrest in Human Colon Cancer Cell Lines. Toxicol. Sci. 2005, 83, 126-135. [CrossRef] [PubMed]

24. Wiesner, M.; Schreiner, M.; Glatt, H. High mutagenic activity of juice from pak choi (Brassica rapa ssp. chinensis) sprouts due to its content of 1-methoxy-3-indolylmethyl glucosinolate, and its enhancement by elicitation with methyl jasmonate. Food Chem. Toxicol. 2014, 67, 10-16. [CrossRef] [PubMed]

25. Agerbirk, N.; Vos, M.D.; Kim, J.H.; Jander, G. Indole glucosinolate breakdown and its biological effects. Phytochem. Rev. 2008, 8, 101-120. [CrossRef]

26. Vig, A.P.; Rampal, G.; Thind, T.S.; Arora, S. Bio-protective effects of glucosinolates-A review. LWT Food Sci. Technol. 2009, 42, 1561-1572. [CrossRef]

27. Ku, K.M.; Jeffery, E.H.; Juvik, J.A. Influence of Seasonal Variation and Methyl Jasmonate Mediated Induction of Glucosinolate Biosynthesis on Quinone Reductase Activity in Broccoli Florets. J. Agric. Food Chem. 2013, 61, 9623-9631. [CrossRef]

28. Ku, K.M.; Juvik, J.A. Environmental Stress and Methyl Jasmonate-mediated Changes in Flavonoid Concentrations and Antioxidant Activity in Broccoli Florets and Kale Leaf Tissues. HortScience 2013, 48, 996-1002. [CrossRef]

29. Clarke, D.B. Glucosinolates, structures and analysis in food. Anal. Methods 2010, 2, 310-325. [CrossRef]

30. Chiu, Y.-C.; Juvik, J.A.; Ku, K.-M. Targeted Metabolomic and Transcriptomic Analyses of "Red Russian" Kale (Brassicae napus var. pabularia) Following Methyl Jasmonate Treatment and Larval Infestation by the Cabbage Looper (Trichoplusia ni Hübner). Int. J. Mol. Sci. 2018, 19, 1058. [CrossRef]

31. Kim, M.J.; Chiu, Y.-C.; Kim, N.K.; Park, H.M.; Lee, C.H.; Juvik, J.A.; Ku, K.-M. Cultivar-Specific Changes in Primary and Secondary Metabolites in Pak Choi (Brassica Rapa, Chinensis Group) by Methyl Jasmonate. Int. J. Mol. Sci. 2017, 18, 1004. [CrossRef]

32. Pfalz, M.; Mukhaimar, M.; Perreau, F.; Kirk, J.; Hansen, C.I.C.; Olsen, C.E.; Agerbirk, N.; Kroymann, J. Methyl Transfer in Glucosinolate Biosynthesis Mediated by Indole Glucosinolate $O$-Methyltransferase 5. Plant Physiol. 2016, 172, 2190-2203. [CrossRef]

33. Yu, J.; Zhao, M.; Wang, X.; Tong, C.; Huang, S.; Tehrim, S.; Liu, Y.; Hua, W.; Liu, S. Bolbase: A comprehensive genomics database for Brassica oleracea. BMC Genom. 2013, 14, 664. [CrossRef] [PubMed]

34. Kusznierewicz, B.; Iori, R.; Piekarska, A.; Namieśnik, J.; Bartoszek, A. Convenient identification of desulfoglucosinolates on the basis of mass spectra obtained during liquid chromatography-diode array-electrospray ionisation mass spectrometry analysis: Method verification for sprouts of different Brassicaceae species extracts. J. Chromatogr. A 2013, 1278, 108-115. [CrossRef]

35. Kim, M.J.; Chiu, Y.-C.; Ku, K.-M. Glucosinolates, Carotenoids, and Vitamins E and K Variation from Selected Kale and Collard Cultivars. J. Food Qual. 2017, 2017, 1-8. [CrossRef]

36. Lisec, J.; Schauer, N.; Kopka, J.; Willmitzer, L.; Fernie, A.R. Gas chromatography mass spectrometry-based metabolite profiling in plants. Nat. Protoc. 2006, 1, 387-396. [CrossRef] [PubMed]

37. Ku, K.M.; Jeffery, E.H.; Juvik, J.A.; Kushad, M.M. Correlation of quinone reductase activity and allyl isothiocyanate formation among different genotypes and grades of horseradish roots. J. Agric. Food Chem. 2015, 63, 2947-2955. [CrossRef] [PubMed]

38. He, L.; Diedrich, J.; Chu, Y.-Y.; Yates, J.R. Extracting accurate precursor information for tandem mass spectra by rawconverter. Anal. Chem. 2015, 87, 11361-11367. [CrossRef]

39. Chong, J.; Wishart, D.S.; Xia, J. Using MetaboAnalyst 4.0 for Comprehensive and Integrative Metabolomics Data Analysis. Curr. Protoc. Bioinform. 2019, 68, e86. [CrossRef]

40. Chong, J.; Soufan, O.; Li, C.; Caraus, I.; Li, S.; Bourque, G.; Wishart, D.S.; Xia, J. MetaboAnalyst 4.0: Towards more transparent and integrative metabolomics analysis. Nucleic Acids Res. 2018, 46, W486-W494. [CrossRef] [PubMed] 
41. Sarvan, I.; Verkerk, R.; van Boekel, M.; Dekker, M. Comparison of the degradation and leaching kinetics of glucosinolates during processing of four Brassicaceae (broccoli, red cabbage, white cabbage, Brussels sprouts). Innov. Food Sci. Emerg. Technol. 2014, 25, 58-66. [CrossRef]

42. Hanschen, F.S.; Brüggemann, N.; Brodehl, A.; Mewis, I.; Schreiner, M.; Rohn, S.; Kroh, L.W. Characterization of Products from the Reaction of Glucosinolate-Derived Isothiocyanates with Cysteine and Lysine Derivatives Formed in Either Model Systems or Broccoli Sprouts. J. Agric. Food Chem. 2012, 60, 7735-7745. [CrossRef]

43. Hwang, E.-S.; Kim, G.-H. Effects of various heating methods on glucosinolate, carotenoid and tocopherol concentrations in broccoli. Int. J. Food Sci. Nutr. 2013, 64, 103-111. [CrossRef] [PubMed]

44. Song, L.; Thornalley, P.J. Effect of storage, processing and cooking on glucosinolate content of Brassica vegetables. Food Chem. Toxicol. 2007, 45, 216-224. [CrossRef] [PubMed]

45. Cheong, J.-J.; Choi, Y.D. Methyl jasmonate as a vital substance in plants. Trends Genet. 2003, 19, 409-413. [CrossRef]

46. Cao, S.; Zheng, Y.; Wang, K.; Rui, H.; Tang, S. Effect of methyl jasmonate on cell wall modification of loquat fruit in relation to chilling injury after harvest. Food Chem. 2010, 118, 641-647. [CrossRef]

47. Boonyaritthongchai, P.; Chimvaree, C.; Buanong, M.; Uthairatanakij, A.; Jitareerat, P. Effect of Methyl Jasmonate on Physical and Chemical Properties of Mango Fruit cv. Nam Dok Mai. Horticulturae 2016, 3, 18. [CrossRef]

48. Napoleão, T.A.; Soares, G.; Vital, C.E.; Bastos, C.; Castro, R.; Loureiro, M.E.; Giordano, A. Methyl jasmonate and salicylic acid are able to modify cell wall but only salicylic acid alters biomass digestibility in the model grass Brachypodium distachyon. Plant Sci. 2017, 263, 46-54. [CrossRef]

49. Tian, S.; Liu, X.; Lei, P.; Zhang, X.; Shan, Y. Microbiota: A mediator to transform glucosinolate precursors in cruciferous vegetables to the active isothiocyanates. J. Sci. Food Agric. 2018, 98, 1255-1260. [CrossRef]

50. Liou, C.S.; Sirk, S.J.; Diaz, C.A.C.; Klein, A.P.; Fischer, C.R.; Higginbottom, S.K.; Erez, A.; Donia, M.S.; Sonnenburg, J.L.; Sattely, E.S. A Metabolic Pathway for Activation of Dietary Glucosinolates by a Human Gut Symbiont. Cell 2020, 180, 717-728. [CrossRef] [PubMed]

51. Fuentes, F.; Paredes-Gonzalez, X.; Kong, A.-N.T. Dietary Glucosinolates Sulforaphane, Phenethyl Isothiocyanate, Indole-3-Carbinol/3,3'-Diindolylmethane: Antioxidative Stress/Inflammation, Nrf2, Epigenetics/Epigenomics and In Vivo Cancer Chemopreventive Efficacy. Curr. Pharm. Rep. 2015, 1, 179-196. [CrossRef] [PubMed]

52. Stephensen, P.U.; Bonnesen, C.; Schaldach, C.; Andersen, O.; Bjeldanes, L.F.; Vang, O. N-Methoxyindole-3-Carbinol Is a More Efficient Inducer of Cytochrome P-450 1A1 in Cultured Cells Than Indol-3-Carbinol. Nutr. Cancer 2000, 36, 112-121. [CrossRef] [PubMed]

53. Hanschen, F.S.; Kaufmann, M.; Kupke, F.; Hackl, T.; Kroh, L.W.; Rohn, S.; Schreiner, M. Brassica vegetables as sources of epithionitriles: Novel secondary products formed during cooking. Food Chem. 2018, 245, 564-569. [CrossRef] [PubMed]

54. Chiu, Y.-C.; Matak, K.; Ku, K.-M. Methyl jasmonate treated broccoli: Impact on the production of glucosinolates and consumer preferences. Food Chem. 2019, 299, 125099. [CrossRef] [PubMed]

55. Dosz, E.B.; Jeffery, E.H. Commercially produced frozen broccoli lacks the ability to form sulforaphane. J. Funct. Foods 2013, 5, 987-990. [CrossRef]

56. Havko, N.E.; Major, I.T.; Jewell, J.B.; Attaran, E.; Browse, J.; Howe, G.A. Control of Carbon Assimilation and Partitioning by Jasmonate: An Accounting of Growth-Defense Tradeoffs. Plants 2016, 5, 7. [CrossRef]

57. Nishimura, T.; Kato, H. Taste of free amino acids and peptides. Food Rev. Int. 1988, 4, 175-194. [CrossRef]

58. Beck, T.K.; Jensen, S.; Bjoern, G.K.; Kidmose, U. The Masking Effect of Sucrose on Perception of Bitter Compounds in Brassica Vegetables. J. Sens. Stud. 2014, 29, 190-200. [CrossRef]

59. Chadwick, M.; Gawthrop, F.; Michelmore, R.W.; Wagstaff, C.; Methven, L. Perception of bitterness, sweetness and liking of different genotypes of lettuce. Food Chem. 2016, 197, 66-74. [CrossRef]

(C) 2020 by the authors. Licensee MDPI, Basel, Switzerland. This article is an open access article distributed under the terms and conditions of the Creative Commons Attribution (CC BY) license (http://creativecommons.org/licenses/by/4.0/). 